\title{
Modelos y Teorías en la ciencia del cuidado. Aclaraciones epistemológicas
}

\section{Models and Theories in the science of care. Epistemological clarifications}

\section{Modelos e teorias na ciência do cuidado. Esclarecimentos epistemológicos}

\section{Lucía Federico ${ }^{1}$}

${ }^{1}$ Doctora en Historia y Filosofía de la Ciencia. Licenciada en Ciencias Biológicas. Investigadora del Centro de Estudios de Filosofía e Historia de la Ciencia de la Universidad Nacional de Quilmes

(CEFHIC-UNQ). Profesora de la materia Epistemología de la Enfermería, Licenciatura en Enfermería, Universidad Nacional de Quilmes. Correo electrónico: luciafed@hotmail.com.

Cómo citar este artículo en edición digital: Federico, L. (2020). Modelos y Teorías en la ciencia del cuidado. Aclaraciones epistemológicas. Cultura de los Cuidados (Edición digital), 24 (56) Recuperado de http://dx.doi.org/10.14198/cuid.2020.56.21

Correspondencia: Pacheco 2107, depto. $1^{\circ}$ C. Código postal 1431, Ciudad Autónoma de Buenos Aires, Argentina. Correo electrónico de contacto: luciafed@hotmail.com

Recibido:17/11/2019 Aceptado:21/02/2020

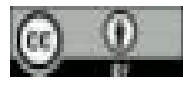

El siguiente artículo fue elaborado en el marco del PICT-2014-1741 (ANPCyT, Argentina), UBACyT-20020170200106BA, UNTREF-32/15 255 y del proyecto "Sobre la naturaleza de la ciencia enfermera. Cerrando la brecha entre teoría y práctica”, en el Programa "Filosofía e Historia de la Ciencia, Etapa II" (PUNQ, 0990/19).

\section{ABSTRACT}

Currently nursing seeks its consolidation as a theoretical scientific field, that of "care", distancing itself from the socalled biomedical sciences. And although, for some time now, nursing theorists have approached the analysis of their disciplinary status, they have not yet reached an agreement, which means that the discussions multiply from the different conceptions of the philosophy of science. Among the theoretical-philosophical articles published in high-impact nursing journals, a work that seeks to shed light is highlighted, using as an

\begin{abstract}
epistemological framework both Kuhnian notions and those of metatheoretical structuralism. The present work analyzes the use of concepts such as "paradigm / disciplinary matrix", "model", "theory" and its adaptation in the aforementioned work, trying to clarify those aspects that are still obscure.
\end{abstract}

Keywords: Epistemology, theories, models, metatheoretical structuralism. 
RESUMEN

En la actualidad la enfermería busca su consolidación como un campo científico teórico, el del “cuidado”, distanciándose de las llamadas ciencias biomédicas. Y si bien, desde hace un tiempo, los teóricos de la enfermería abordan el análisis de su estatus disciplinar, aún no han arribado a un acuerdo, lo que genera que las discusiones se multipliquen desde las distintas concepciones de la filosofía de la ciencia. Entre los artículos teóricos-filosóficos publicados en revistas de enfermería de alto impacto se destaca un trabajo que trata de arrojar luz, utilizando como marco epistemológico tanto nociones kuhnianas como aquellas propias del estructuralismo metateórico. El presente trabajo analiza el uso de conceptos tales como "paradigma /matriz disciplinar”, “modelo”, “teoría” y su adecuación en el mencionado trabajo, tratando de clarificar aquellos aspectos aún oscuros.

Palabras clave: Epistemología, teorías, modelos, estructuralismo metateórico.

\section{RESUMO}

Atualmente, a enfermagem busca sua consolidação como campo científico teórico, o de "cuidar", distanciando-se das chamadas ciências biomédicas. E embora, por algum tempo, os teóricos da enfermagem tenham abordado a análise de seu status disciplinar, eles ainda não chegaram a um acordo, o que significa que as discussões se multiplicam a partir das diferentes concepções da filosofia da ciência. Dentre os artigos teórico-filosóficos publicados em periódicos de enfermagem de alto impacto, destaca-se um trabalho que busca esclarecer, utilizando como referencial epistemológico tanto as noções kuhnianas quanto as do estruturalismo metateórico. O presente trabalho analisa o uso de conceitos como "paradigma / matriz disciplinar", "modelo", "teoria" e sua adaptação no trabalho supracitado, procurando esclarecer os aspectos ainda obscuros.

Palavras-chave: Epistemologia, teorias, modelos, estruturalismo metateórico.

\section{INTRODUCCION}

Cada vez se hace más marcada la demanda de los teóricos de la enfermería de un instrumental elucidatorio, proveniente desde la filosofía de la ciencia, que les permita clarificar tanto cuestiones acerca de su estatus disciplinar como dirimir la naturaleza de sus unidades epistémicas. Esta necesidad ha sido señalada por autores, tanto de habla hispana, como de habla portuguesa e inglesa (ver, por ejemplo, Siles González, 2016; Carvalho, 2003; Risjord, 2010). Lo recurrente es que en el área se haga uso para tal fin las filosofías de la ciencia tradicionales (el empirismo lógico o el hipotético-deductivismo de Popper) o del enfoque dinámico de Kuhn o de alguna tradición por fuera de la filosofía analítica, como por ejemplo la fenomenología u otra corriente interpretativista. Sin embargo hay algunos artículos que, aunque contados, se salen de lo común y asombran con propuestas elucidatorias más modernas. Ese es el caso del artículo de Salas Iglesias (2003) publicado en esta revista, el cual realizó su aporte desde el Estructuralismo Metateórico, una propuesta relevante enmarcada dentro de la familia semanticista de la filosofía de la ciencia. Y si bien festejamos su iniciativa, creemos que la adecuación epistemológica del texto en cuestión puede ser refinado a todo fin útil. 
Por tanto, el presente trabajo pretende analizar el uso de conceptos tales como “paradigma /matriz disciplinar”, “modelo” y “teoría” de la señalada publicación y su adecuación al área de la enfermería, tratando de clarificar aquellos aspectos aún oscuros. Vale la pena el esfuerzo en cuestión, pues el trabajo mencionado es original respecto a las discusiones actuales sobre el conocimiento enfermero, cuestión que resulta imprescindible clarificar para mejorar la formación teórica de sus practicantes.

\section{TEORÍA Y MÉTODO. EL ESTATUS}

DE LA ENFERMERÍA: PARADIGMA, MÉTODO Y NATURALEZA DE SU OBJETO DE ESTUDIO

El objetivo del señalado artículo, en palabras del autor, es "el de tratar de definir cuál es la situación actual del estatus científico de la enfermería, así como distinguir los principales problemas que impiden su consolidación en el contexto general de la ciencia” (Salas Iglesias, 2003: 71). En busca de una respuesta, el autor decide analizar, por un lado, las unidades de ciencia de la enfermería, y, por el otro, su método. En el presente trabajo dejaremos de lado el segundo de los puntos por haberlo discutido ya en Federico (2017). Por tanto nos concentraremos en el primero de ellos, es decir, en el análisis sobre las unidades de ciencia (estratificadas) de la enfermería, cuyo origen radica en la propuesta canónica de Jacqueline Fawcett, de uso común en la bibliografía del área. La estructura jerárquica de Fawcett consiste en una "holarquía” (2005, p.3) que va de lo más abstracto a lo más empírico. El primero de los concepto, y el de mayor grado de abstracción, es el de metaparadigma. Este concepto fue introducido en la enfermería en los años setenta y si bien Fawcett no fue la primera en usarlo fue la que lo popularizo en 1984. Le siguen, con un grado menor de abstracción, los modelos conceptuales o modelos. Y si bien el primer modelo desarrollado puede ser considerado el de Nightingale (1855), la etiqueta "modelo" se hizo explícita a partir de la mitad de los años setenta. Luego, los elementos de menor generalidad que le sigue son las teorías. Las teorías pueden variar en cuanto su grado de abstracción: las mas abstractas son las “teorías generales”, que se derivan, según la autora, directamente de los modelos conceptuales, mientras que las más concretas y restringidas son las "teorías de rango-medio”.

A continuación, se presentan los puntos más relevantes de la elucidación de Salas Iglesias al respecto.

El estado actual de la "ciencia enfermera", afirma Salas Iglesias, debe ser evaluado tomando en cuenta los siguientes indicadores:

El inconcluso debate respecto a la definición misma de la Enfermería, la definición imprecisa de su objeto de estudio y la determinación de su naturaleza, las reificantes consecuencias del uso indistinto $e$ 
Revista científica de la Asociación de Historia y Antropología de los Cuidados (Universidad de Alicante)

indiscriminado de las nociones de enfermería, cuidado y rol profesional [...]; así como la vaguedad en los planteamientos respecto a si la enfermería está más cerca de las ciencias sociales y humanas que de las fácticas o naturales (2003: 71-72).

Sorteando la distinción entre ciencias sociales o humanísticas y las naturales (ambas fácticas, de acuerdo a la distinción clásica de Bunge, 2005), del diagnostico se desprende que los temas que ocupan al conflictuado campo disciplinar de la enfermería, centro de "los esfuerzos disciplinares”, son: I. la definición de la naturaleza de su objeto de estudio, II. la historia de la formación de sus conceptos y teorías y III. "la ausencia de posicionamientos epistemológicos” adecuados para su resolución.

En un primer acercamiento a la clarificación de los problemas planteados, el autor hace uso de La estructura de las revoluciones científicas de T.S. Kuhn, en la versión posterior al '62 que incluye $L a$ posdata. Salas Iglesias afirma entonces, sin tener en cuenta las advertencias de Kuhn incluidas en dicha posdata, que la situación descripta por él acerca la enfermería coincide con la fase de “preciencia”(también llamada preparadigmática):

Siendo este estadio perfectamente reconocible en la situación actual de la enfermería, donde uno de los principales síntomas de los que adolecen sus profesionales, es la casi generalizada necesidad de definir una teoría propia a la hora de establecer su marco de intervención, más allá de los modelos teóricos existentes. Lo que, por otra parte, evidencia la ausencia de modelos efectivos en nuestro ámbito (2003: 73).

Cuando Salas Iglesias señala la falta de "modelos efectivos" no usa el termino "modelo" ni de forma coloquial ni de la forma en que la utilizan comúnmente los científicos, sino que más bien apunta a un uso particular que se entenderá más adelante en el desarrollo de su texto, pero que advertimos merece especial atención. El autor, que continúa presentando las características que distinguen "ciencia normal” de "preciencia”, advierte que "para Kuhn, será justamente la existencia de un paradigma que pueda apoyar una tradición de ciencia normal lo que establecerá la diferencia entre lo que es Ciencia [mayúscula del autor] y lo que no lo es” (2003: 73). Pese a esta tajante distinción que el autor hace de las palabras de Kuhn, luego, en un tono más moderado, considera a sendos momentos de la dinámica kuhniana de la ciencia como "ciencia madura" y “ciencia inmadura”, por lo que esta última ya no es tratada como “ausencia de ciencia”. Para el diagnóstico del estatus disciplinar de la enfermería, Salas Iglesias se enfoca en uno de los problemas antes señalados, a 
saber, el de las unidades de ciencia, para lo cual analiza la noción kuhniana de “paradigma”.

Un paradigma, siguiendo a Salas Iglesias, permite el desarrollo de un conjunto de teorías bajo una cosmovisión, por lo que propone la posibilidad de asimilar la noción de "metaparadigma" introducida por Fawcett en los '80 (2005) con la de “paradigma”:

No nos encontraríamos lejos de hallar, en esta exposición, la explicación de lo que por metaparadigma entendemos respecto a nuestra disciplina, por cuanto éste viene a suponer una serie de conceptos (cuidado, persona, salud, entorno) que se articulan constituyendo proposiciones que le atribuyen su propia especificidad y, a raíz de las cuales, se originan teorías, aunque en nuestro caso es mejor hablar, como venimos participando, de modelos potenciales (2003: 73).

La utilización de "modelos potenciales” aquí tampoco es casual. Y si bien Salas Iglesias propone el concepto de “paradigma” para referirse al “metaparadigma” de Fawcett, de todos modos, considera la "ciencia enfermera” como ciencia inmadura por sus características actuales:

los conceptos carecen de nivel de abstracción suficiente, sobre todo el cuidado, como para desvincularse de las limitaciones características del reduccionismo de la ideología científica racional-tecnológica. Por consiguiente, las proposiciones que de sus relaciones se deriven, lo que vienen a demostrar, es una gran incapacidad para responder a los interrogantes de nuestro universo de estudio. [...]. Luego, todo lo que de aquí se derive no va a construir en grado alguno al desarrollo disciplinar, sino todo lo contrario (2003: 73).

El autor usa el término “ideología” de forma ambigua. Por lo que se entiende a lo largo de su texto, Salas Iglesias refiere a una tradición epistemológica, pues menciona al positivismo, la teoría crítica y la hermenéutica (un análisis al respecto se puede ver en Minguez-Moreno \& Siles González, 2014). Su crítica apunta a que la “ideología” usual, encarnaría una racionalidad particular que define “eficiencia” y otros criterios meramente técnicos como aquellos necesarios $\mathrm{y}$ suficientes para las decisiones y por ende para el progreso, dejando fuera nociones de cuidado menos “tecnológicas” pero aceptadas en la enfermería. Entre estas nociones donde la eficiencia no sería el valor supremo, creemos que el autor refiere a una noción más empática con el paciente. El resultado de esto, según Salas Iglesias, es contundente: “Esta situación estructural define, por si misma, el estatus científico en el que nos encontramos que, si algo evidencia, es precisamente la 
Revista científica de la Asociación de Historia y Antropología de los Cuidados (Universidad de Alicante)

ausencia de ciencia consolidada” (2003:

72).

Ahora bien, el autor advierte que la demanda de un "monoparadigma" viene tradicionalmente de la mano de una ciencia enfermera adscrita a las ciencias naturales, desde cuyo marco se piensa a la enfermería en el mundo anglosajón, pero no ocurre igual "en las ciencias sociales, cuya naturaleza es multiparadigmática” (2003: 74). Y si bien, nuestro autor no toca el punto sobre la historia de sus unidades de ciencia, sí analiza desde dónde se ha pretendido responder a las cuestiones fundamentales de la enfermería y que, según el autor, aún se encuentran estrechamente vinculadas con el pensar la enfermería como parte de las ciencias naturales: “cuya ideología científica dominante y hegemónica a lo largo de la historia de la filosofía de la ciencia, ha sido el positivismo" (2003: 73). Suponemos que el autor se refiere al positivismo comteano, $\mathrm{y}$ no al positivismo lógico del círculo de Viena, o al menos no al que surge del análisis profundo del mismo, lejos de la versión estándar que lo distorsionó por sostener una ideología política de izquierdas (ver Gómez, 2014). Pero si la ciencia enfermera se encuentra más próxima a las ciencias sociales, afirma el autor, "no podemos negarle el carácter de ciencia normal a la enfermería arguyendo que no se rige por un único paradigma o ideología científica, toda vez que queda demostrada su naturaleza compleja” (2003: 74).
En síntesis, si el análisis epistemológico del estatus actual de la enfermería viene de la mano de una concepción afín con las ciencias naturales, la enfermería, demarcada por el metaparadigma, es ciencia inmadura, pues aún hay desacuerdos en cuanto la cosmovisión y el método (lo que es articulado como ausencia de un “monoparadigma” o paradigma único). Caso contrario es el aceptar que es una ciencia social $\mathrm{y}$, aunque madura, contiene una multiplicidad de unidades de ciencia que explica las diferentes cosmovisiones (es decir, es multiparadigmática) y, por tanto, conviven en su seno diversos desacuerdos. Pero no queda claro entonces que se va a entender por "metaparadigma”.

Bajo este enfoque que consiste en pensar la enfermería como parte de las ciencias sociales, bastante extendido entre los teóricos actuales de la enfermería, Salas Iglesias se pregunta: ¿qué es entonces un paradigma y cómo se articula la realidad expuesta para la enfermería?

En busca de respuestas retoma la crítica de Margaret Masterman (1970/1975) a La estructura de Kuhn, tal como antes lo hizo en su momento Fawcett (2005: 4). Es interesante destacar que Fawcett toma la noción de "metaparadigama" de una de las propuestas efectuadas en el legendario texto de Masterman sobre los veintiún significados de paradigma de Kuhn:

La idea para un componente de conocimiento llamado metaparadigma 
Cultura de los Cuidados

surge en la discusión de los múltiples significados que Kuhn (1962) le ha dado al termino paradigma. Masterman (1970) señaló que un significado refleja la metafísica más que la noción científica de entidad, etiqueta que significan el metaparadigma (Fawcett, 2005:4).

Fawcett hace referencia a uno de los significados que encuentra Masterman de paradigma, vinculado al significado numero 8, a saber, "como especulación metafísica acertada":

Sin embargo Salas Iglesias descarta la noción de "paradigma" alla Masterman elegida por Fawcett (“paradigma” como un conjunto de creencias o marco de referencia) por entender que su noción de “metaparadigma” porta creencias afines al espíritu del positivismo tan rechazable para el autor:

Por consiguiente, cuando desde la enfermería hablamos del "metaparadigma" como el marco de referencia que encierra las respuestas a las cuestiones fundamentales de la disciplina, se evidencia que su contenido responde a los criterios de creación y desarrollo científico de la ideología racional-tecnológica que, como venimos introduciendo, resulta inadecuada a su naturaleza (2003: 75). El autor no nos dice cómo, a partir del monismo metodológico, de la racionalidad instrumental y de la ciencia monoparadigmática se sigue la afirmación anterior.
Salas Iglesias decide luego hacerse eco de otro de los sentidos de "paradigma" propuestos por Masterman (que luego retomará Kuhn en su reformulación de la noción en la Posdata a La estructura): “en aras a la comprensión metodológica y al ideológico uso neutral de los términos, resulta apropiado introducir un nuevo concepto: la matriz disciplinar" (2003: 75). Entonces interpretamos que el autor propone "paradigma” como la unidad epistemológica de conocimiento más abstracta (lo que desde Fawcett llamamos “metaparadigma”), reservando "matriz disciplinar”, concepto menos abstracto, como unidad de ciencia. En palabras de Salas Iglesias:

En resumen, mediante el concepto de paradigma se pretende dar a conocer una cierta unidad científica, metodológica y sociológica que tiene lugar en un momento histórico concreto, donde la matriz disciplinar debe verse como una entidad, en donde sus elementos dan forma al paradigma, libre del resto de significaciones que se le atribuyen a dicho concepto, refiriendo a los contenidos mismos de la filosofía de la ciencia (2003: 75).

Entonces nos podemos preguntar: ¿matriz disciplinar es algo así como algún sentido de “teoría”? Algo que también preocupa al autor pues para que la noción kuhniana sea aplicada correctamente, advierte, hace falta distinguir, en primer lugar “paradigma” y “teoría”, 
Revista científica de la Asociación de Historia y Antropología de los Cuidados (Universidad de Alicante)

...por cuanto de entre los diversos usos que de paradigma se ha venido a hacer, la comunidad científica enfermera ha participado de una de sus acepciones en tanto "uno de los niveles de desarrollo de la teoría". Efectivamente la comunidad científica dirá que comparte una teoría (2003: 75)

Ello para el autor implica, con mayor precisión, compartir una misma matriz disciplinar. Pero a su vez advierte que es así, siempre y cuando ésta supone de dotar a la disciplina de un conjunto de elementos, que deben orientar el proyecto de construcción científico respecto a la filosofía de la ciencia. Y, en ese sentido, las teorías refieren al área lógica del citado contexto (2003: 78).

Advierte también que hace falta distinguir “teoría” de “modelo”:la realidad de la situación teórica de la enfermería exige, en primera instancia, hacer la distinción entre teoría y modelo, por cuanto tal diferenciación responde a exigencias de tipo lógico, siendo ésta quien estudia la estructura interna y dinámica de las teorías científicas (2003: 76).

Con la convicción de tomar un “posicionamiento epistemológico adecuado” Salas Iglesias propone para dicha distinción el uso del Estructuralismo Metateórico, citando así a dos de sus autores canónicos, los filósofos Joseph Sneed y Wolfgang Stegmüller. Asimismo, pretende ofrecer una visión más clara de por qué se dan los problemas antes señalados (como el poco grado de abstracción, la confusión en el uso de modelos y teorías, etc.) en la ciencia enfermera. A continuación, haremos referencia a la presentación que Salas Iglesias hace del instrumental metateórico estructuralista:

Desde el estructuralismo de Sneed y Stegmüller, se postula que la teoría general de una ciencia particular está constituida por un núcleo y por sus aplicaciones o área de aplicabilidad (aplicación intencional). Del mismo modo, este núcleo está constituido por una serie de componentes, entre los que destacamos los modelos teóricos. Estos pueden ser Efectivos (engloba todos los ámbitos empíricos en los que la teoría resulta ser verdadera), Potenciales (aquellos que podrían ser modelo de la teoría o que, aún siéndolos, todavía no han sido contrastados como efectivos) o Parciales (2003: 76).

Luego presenta las condiciones de ligadura y vínculos interteóricos y continúa:

Se puede afirmar que la ciencia es una red. Tras esta estructura matemática del núcleo se encuentra la parte pragmática, entendida como la comprobación de su efectividad. Es decir, en toda teoría hay una componente pragmática, que nos lleva a distinguir quién, en qué momento y en qué condiciones consideró su modelo potencial y comprobó si era efectivo o no. Constituye, pues, un condicionante de toda teoría científica (2003: 76). 
Determina así que "nos encontramos en condiciones de afirmar que la producción teórica enfermera, más que a la definición de “teoría de la enfermería”, refiere básicamente al concepto de "modelos potenciales"” (2003: 76). De allí se entiende la afirmación anterior del autor de que en enfermería faltan "modelos efectivos", algo como teorías con aplicación exitosa. Lo que habría pues son "modelos potenciales”, algo como... ¿teorías aún sin aplicaciones exitosas?

Así parece que Salas Iglesias da cuenta del por qué de la inmadurez del estatus disciplinar de la enfermería, ya que ésta no logra aún aplicaciones exitosas de sus marcos conceptuales mas abstractos 0 matrices disciplinares, que aún están (apenas) en un estado de "modelos potenciales" que proliferan y proliferan pero que ninguno de ellos se torna o es "efectivo" (o muy pocos lo hacen, pues el propio autor menciona al pasar algunas teorías exitosas), lo que se hace eco respecto a lo complejo de su objeto de estudio, que ninguna noción previa captura por completo.

\section{DISCUSIÓN}

Sobre el estatus de la madurez de la ciencia enfermera no creemos que haya mucho más que señalar que lo ya señalado por la misma Margaret Masterman:

Hay, sin embargo, un profundo contraste entre este estado de cosas precientífico y filosófico y la ciencia

con múltiples paradigmas, ese otro estado de cosas en el que, lejos de no haber ningún paradigma, hay por contrario, demasiados (este es el estado general actual de las ciencias psicológicas, las ciencias sociales y las ciencias de la información). Aquí, dentro del subdominio definido por cada técnica paradigmática, la tecnología puede algunas veces llegar a estar muy avanzada, y la investigación normal de resolución de enigmas puede realizar progresos (1970/1975: 180).

Y luego Kuhn en La posdata:

La naturaleza de esa transición a la madurez merece un análisis más complejo del que ha recibido en este libro [en su versión original de 1962], particularmente de aquellos interesados en el avance de las ciencias sociales contemporáneas. [...]. Los miembros de todas las comunidades científicas, incluso de las escuelas del período "preparadigmático" comparten las clases de elementos que, colectivamente, he llamado un "paradigma". Lo que cambia con la transición a la madurez no es la presencia de un paradigma, sino, antes bien, su naturaleza (1969: 274-275).

En definitiva, la naturaleza multiparadigmática actual de la ciencia enfermera no difiere de la de otras disciplinas científicas cuyo estatus no está sujeto a polémicas, como el caso de la 
Revista científica de la Asociación de Historia y Antropología de los Cuidados (Universidad de Alicante)

sociología o la biología evolutiva, por lo que creemos que aquello relevante a analizar no pasa por este punto. Tampoco resulta de mayor utilidad discutir sobre la inscripción de la enfermería dentro de las ciencias fácticas naturales o sociales. Más bien lo relevante será elucidar la naturaleza de sus unidades epistemológicas de análisis. Retomaremos, por tanto, algunas nociones filosóficas discutidas por Fawcett y Salas Iglesias acerca de las unidades de ciencia enfermera. Para ello, debemos recapitular las reflexiones de Masterman que sirve a ambos como marco de referencia.

Los tres sentidos que propone Masterman de "paradigma”, al condensar los veintiún sentidos encontrados en el libro de Kuhn, son, en primer lugar, aquel asimilable a "metaparadigma" ya introducido en la sección anterior. En segundo lugar, el sentido sociológico, de hábitos compartidos por los científicos, denominado "paradigma sociológico”. En tercer lugar, el "paradigma artefacto" de resolución de enigmas o rompecabezas (1970/1975: 169).

Masterman también hace notar que ninguna de las nociones o sentidos de paradigma concuerdan con la noción o sentidos de “teoría” de los filósofos de la ciencia tradicionales, algo con lo que Kuhn acuerda en La posdata: "Los científicos mismos dirán que comparten una teoría o un conjunto de teorías [...]. Sin embargo, "teoría”, tal como se aplica usualmente en la filosofía de la ciencia, connota una estructura mucho más limitada en naturaleza y alcance que la requerida aquí” (1969: 278).

De allí que utiliza, para evitar confusiones, "matriz disciplinar”.

A diferencia de Fawcett que se queda con la primera de las nociones, sumamente criticada por los filósofos y que genera una serie de confusiones y discusiones tanto en el seno de la enfermería como en la filosofía, Salas Iglesias se hace eco de la tercera noción (la de matriz disciplinar), no desde Masterman sino desde La posdata de Kuhn, lo cual nos parece una estrategia adecuada. Pero como ya vimos, descarta la noción de “metaparadigma” bajo una justificación epistemológica desafortunada. Sin embargo, y por las razones que fuera, propone en su lugar usar "paradigma" como "unidad científica, metodológica y sociológica en un momento histórico" (2003: 75) para delimitar la disciplina enfermera. Esto, en definitiva, es lo que Kuhn refiere con la noción de "comunidad científica” y con la cuál acordamos (para otros análisis al respecto ver Badillo Zúñiga, Ostiguín Melénez \& Bermúdez González, 2013; Siles González \& García Hernández, 1995). En palabras de Salas Iglesias:

[paradigma] es un conjunto de supuestos epistemológicos fundamentales, a partir de los cuales una comunidad científica determinada (comunidad epistémica) explica el mundo, o la parte del universo que le interesa (en nuestro caso, el que concierne al cuidado)” (2003: 75).

Acto seguido el autor propone como "unidad de ciencia" la noción kuhniana de "matriz 
disciplinar”, la cual usa además para dar cuenta del carácter “multiparadigmático” de la enfermería. Diferenciándose de Fawcett (2005: 16), que acepta “modelo conceptual” como sinónimo de “matriz disciplinar” y de (creemos) la (primera noción imprecisa kuhniana de) “paradigma”, Salas Iglesias propone que lo que debe ser entendido como “matriz disciplinar” son las "teorías”, no quedando en claro aún el papel de los “modelos”. Pero para resolverlo se plantea una elucidación haciendo uso del instrumental estructuralista. A continuación, nos concentraremos en dicha elucidación.

En su texto Salas Iglesias afirma que la distinción entre “teoría” y “modelo” se debe a su carácter lógico. Al efectuar un análisis con el instrumental estructuralista, se desentiende de la noción de matriz disciplinar y asocia "teoría” con la noción de “modelo potencial” estructuralista: “teorías como modelos potenciales”. La pregunta es, ¿serán estas teorías, en estado de “modelo potencial”, las “matrices disciplinares” de la enfermería?

Quizás esta particular manera de entender "teoría”, "matriz disciplinar” y "modelo potencial” se deba a que Salas Iglesias interpreta "matriz disciplinar" de la siguiente manera:

La matriz de la disciplina viene a comprender la totalidad de los objetos de consenso científico y destaca como sus elementos: las generalizaciones simbólicas (aceptación, por parte de la comunidad científica, de la forma $e$ interpretación lógica de las leyes o ecuaciones fundamentales de las teorías), los modelos (identificación de los problemas no resueltos), los valores (valores epistémicos de las teorías, tales como exactitud, consistencia, simplicidad y beneficio) y las soluciones de problemas típicos que sirven como ejemplos (problemas ejemplares), que es la denominación de paradigma en un sentido estricto (2003: 75).

Nótese que los "modelos" de la matriz disciplinar son la "identificación de los problemas no resueltos”. Sobre esto, en primer lugar, lo que Salas Iglesias entiende por modelo no se corresponde a la noción kuhniana de “modelo”, la cual es descrita en La estructura:

Un segundo tipo de componente de la matriz disciplinar [...] "las partes metafísicas de los paradigmas”. Estoy pensando en compromisos compartidos [...]. Al rescribir el libro describiría yo ahora tales compromisos como creencias en modelos particulares, y extendería los modelos de categorías para que también incluyeran una variedad relativamente heurística. [...]. Desde los modelos heurísticos hasta los ontológicos, todos ellos tienen funciones similares, y entre otras cosas, proporcionan al grupo las analogías y metáforas preferidas o permisibles. Siendo así, ayudan a determinar lo que será aceptado tanto como una 
Revista científica de la Asociación de Historia y Antropología de los Cuidados (Universidad de Alicante)

explicación o como una soluciónenigma (1969: 282-283).

En segundo lugar, parece que Salas Iglesias está entendiendo “identificación de los problemas no resueltos" como “modelos potenciales”, quizás porque (según el Estructuralismo Metateórico) los modelos potenciales, al simbolizar la clase total de entidades de las que da cuenta la teoría, conforman las estructuras de las cuales tiene sentido preguntarse si son modelos (efectivos o actuales), pero que todavía no se sabe si efectivamente lo son. De allí que además diga que la enfermería carece de “modelos efectivos”, es decir de aquellos modelos donde además se cumplan las leyes. Esto puede ser vinculado además con la falta de aplicaciones exitosas, según Salas Iglesias, por lo complejo de su fenómeno de estudio: el cuidado.

Ahora bien, si se acepta la interpretación aquí presentada, entonces podrían entenderse las siguientes palabras del autor: "No obstante, cabría señalar los esfuerzos que la comunidad científica enfermera está realizando por consolidar la parte pragmática de toda teoría científica” (2003: 76). Pues según el instrumental estructuralista toda aplicación o deseo de aplicación de la teoría a un fenómeno particular, el conjunto de las aplicaciones pretendidas, lleva componentes pragmáticos, algo que también cita el autor.
Pese a este intento elucidatorio es poco claro aún cómo encajan en el entramado jerárquico de las unidades de conocimiento de la enfermería los “modelos potenciales”, que, para el estructuralismo, son parte de una teoría, es decir de un elemento teórico (o mínima unidad de ciencia). Algo muy distinto a lo que Kuhn llama "matriz disciplinar” que, para empezar, contiene leyes de distintos niveles de abstracción o de generalidad. Y si bien celebramos la propuesta elucidatoria de Salas Iglesias que, a diferencia de otros autores que no ven más allá de Kuhn, ha avanzando en la búsqueda de alternativas filosóficas contemporáneas, no ha logrado aún una clarificación de las unidades epistémicas de la enfermería. Esto quizás tenga que ver con haber pasado por alto que el Estructuralismo cuenta con una noción más afín a “matriz disciplinar” que le permite capturar la complejidad de las unidades de ciencia: la noción de "red teórica”. Aunque nuestro autor la menciona en su texto, no la usa. Esto resulta más grave aún si tenemos en cuenta que se encuentra a disposición la interpretación kuhniana de dicha noción (Kuhn, 1976), lo que facilita o hace más accesible su aplicación.

\section{CONSIDERACIONES FINALES}

Las palabras de Salas Iglesias nos recuerdan una vez más la necesidad de un adecuado instrumental filosófico para pensar las unidades de ciencia: "La comunidad científica enfermera precisa consolidar su 
Cultura de los Cuidados

acuerdo en lo sociológico y en lo filosófico siempre que su carencia contribuye más a su distorsión que al desarrollo disciplinar" (2003: 77-78).

Si bien el autor hizo su aporte desde un lugar original, el Estructuralismo Metateórico, actualmente no es el único. La conocida historiadora y teórica de la enfermería Hernández Conesa, “recoge el guante” y finaliza su nota editorial del 2006, "La enfermería y el conocimiento científico" con la misma frase de nuestro autor:

Desde ese espacio educativo superior comienza la andadura de procurarse una estructura metodológica, así como estructuras interpretativas a través de los Modelos y Teorías, que si bien, desde la Filosofía de la Ciencia y desde la corriente estructuralista de Sneed y Stegmüller, serían modelos potenciales cuyas aplicaciones intencionales estarán aún limitadas, no deja de ser un camino riguroso en el contexto científico contemporáneo (2006: 7).

Pero, al igual que nuestro autor, se equivoca en el diagnóstico debido a que el instrumental filosófico no fue ni bien usado ni se hizo uso de todo su arsenal y potencial. Pues bien, ante este diagnóstico introduciremos a continuación algunas nociones esenciales del estructuralismo (Balzer, Moulines \& Sneed, 1987). Una de las primeras nociones a mencionar es la de las “clases de modelos" (en conjunto los “modelos potenciales”, “actuales” y “potenciales parciales”), noción que sólo es útil para capturar lo que el estructuralismo entiende por un “elemento teórico”, la estructura más sencilla que permite dar cuenta de una unidad de ciencia. Pero, como ya se mencionó, se queda corta para analizar la complejidad de la noción de "matriz disciplinar” de Kuhn. Ciertamente hubiera sido apropiado, para no perder los niveles de jerarquía de las estructuras enfermeras, hacer uso de la noción de "red teórica" ya mencionada.

Sin embargo, esto no basta, pues lo que hace falta aún para clarificar las unidades de conocimiento, el estatus de cada una y los vínculos entre ellas es el análisis formal de cada unidad, es decir, de los llamados modelos de la enfermería, de las teorías generales y de las teorías de rango medio. Recién cuando se analice la estructura de cada una de ellas se podrán analizar sus vínculos $\mathrm{y}$, recién allí, se podrá tener un diagnostico más claro de la estructura jerárquica propuesta por Fawcett.

Esta tarea que le concierne a la filosofía de la ciencia, que no por ello excluye a los teóricos de la enfermería con manejo del arsenal filosófico, es una tarea claramente distinta a la científica. Se trata de una tarea puramente teórica o conceptual (como lo es la propuesta de holojerarquía de Fawcett), algo que también expresa Salas Iglesias al hablar de la situación actual del campo disciplinar, que:

se caracteriza por confundir, con mayor frecuencia de lo deseable, la forma con el fondo, constituyendo el eje o foco de 
Revista científica de la Asociación de Historia y Antropología de los Cuidados (Universidad de Alicante)

dicho desgaste científico, el

instrumento de intervención en vez de la meta en sí misma. Como resultado, se ha obtenido que la práctica enfermera sea, más bien, consecuencia de un proceso de trabajo (con implicaciones taxonómicas), que de una teoría científica. Sería acertado afirmar que, en ocasiones, los árboles no nos dejan ver el bosque (2003: 76).

Con las acertadas palabras de nuestro autor cerramos el presente trabajo, no por ello dejando de señalar que, si bien aún queda mucho por hacer, ya se comenzó a efectuar el análisis estructuralista de las unidades de ciencia enfermera. Así esperamos obtener prontamente resultados que ayuden a iluminar las penumbras que quedan del bosque.

\section{BIBLIOGRAFÍA}

Badillo Zúñiga, J., Ostiguín Melénez, M.R. y Bermúdez González, A. (2013). El paradigma: un análisis del concepto y su implicación conceptual en enfermería. Revista Iberoamericana de educación en enfermería, 3(2), 18-25.

Balzer, W., Moulines, C.U. \& Sneed, J.D. (1987). An Architecture for Science. The Structuralist Program. Dordrecht: Reidel.

Bunge, M. (2005). La ciencia: su método y su filosofía. Buenos Aires: De Bolsillo.

Carvalho, V. (2003). Acerca de las bases teóricas, filosóficas, epistemológicas de la investigación científica - el caso de la enfermería. Rev Latino-am Enfermagem; 11(6), 807-815.
Fawcett, J. (2005). Contemporary Nursing Knowlwdgw. Analysis and Evaluation of Nursing Models and Theories. Philadelphia: F.A. Davis Company.

Federico, L. (2017). Una mirada filosófica del Proceso de Atención en Enfermería. Aportes a la enseñanza en enfermería. Revista Territorios del Cuidado, 1(2), 63-76. http://sociales.unq.edu.ar/wpcontent/upload s/2017/12/revista_territorios_del_cuidadoCOMBINADA-CON-ISSN.pdf

Gómez, R. (2014). La dimensión valorativa de las ciencias: hacia una filosofía política. Bernal: Universidad de Quilmes.

Hernández Conesa, J. (2006). La enfermería y el conocimiento científico. Enfermería Universitaria, 3(3), 3-7.

Kuhn, T.S. (1962/1992). La estructura de las revoluciones Científicas. España: Fondo de Cultura Económica.

(1976). Theory Change as Structure Change: Comments on the Sneed Formalism. Erkenntnis, 10, 179-199.

Masterman, M. (1970/1975). La ciencia normal y sus peligros, en I. Lakatos, y A. Musgrave (eds.), La crítica y el desarrollo del conocimiento. Barcelona: Grijalbo.

Minguez-Moreno, I., y Siles González J. (2014). Pensamiento crítico en enfermería: de la racionalidad técnica a la práctica reflexiva. Aquichan, 14(4), 594-604.

Risjord, M.W. (2010). Nursing Knowledge. Science, Practice and Philosophy. WileyBlackwell.

Salas Iglesias, M.J. (2003). Teoría y método. El estatus científico de la enfermería: paradigma, método y naturaleza de su objeto de estudio. Cultura de los cuidados, VII(14): 71-78. 
Siles González, J. (2016). La utilidad práctica de la Epistemología en la clarificación de la pertinencia teórica y metodológica en la disciplina enfermera. Index de Enfermería, 25(1-2), 86-92.

Siles González J. y García Hernández E. (1995). Las características de los paradigmas y su adecuación a la investigación en enfermería. Enfermería Científica, 160-161, 10-14

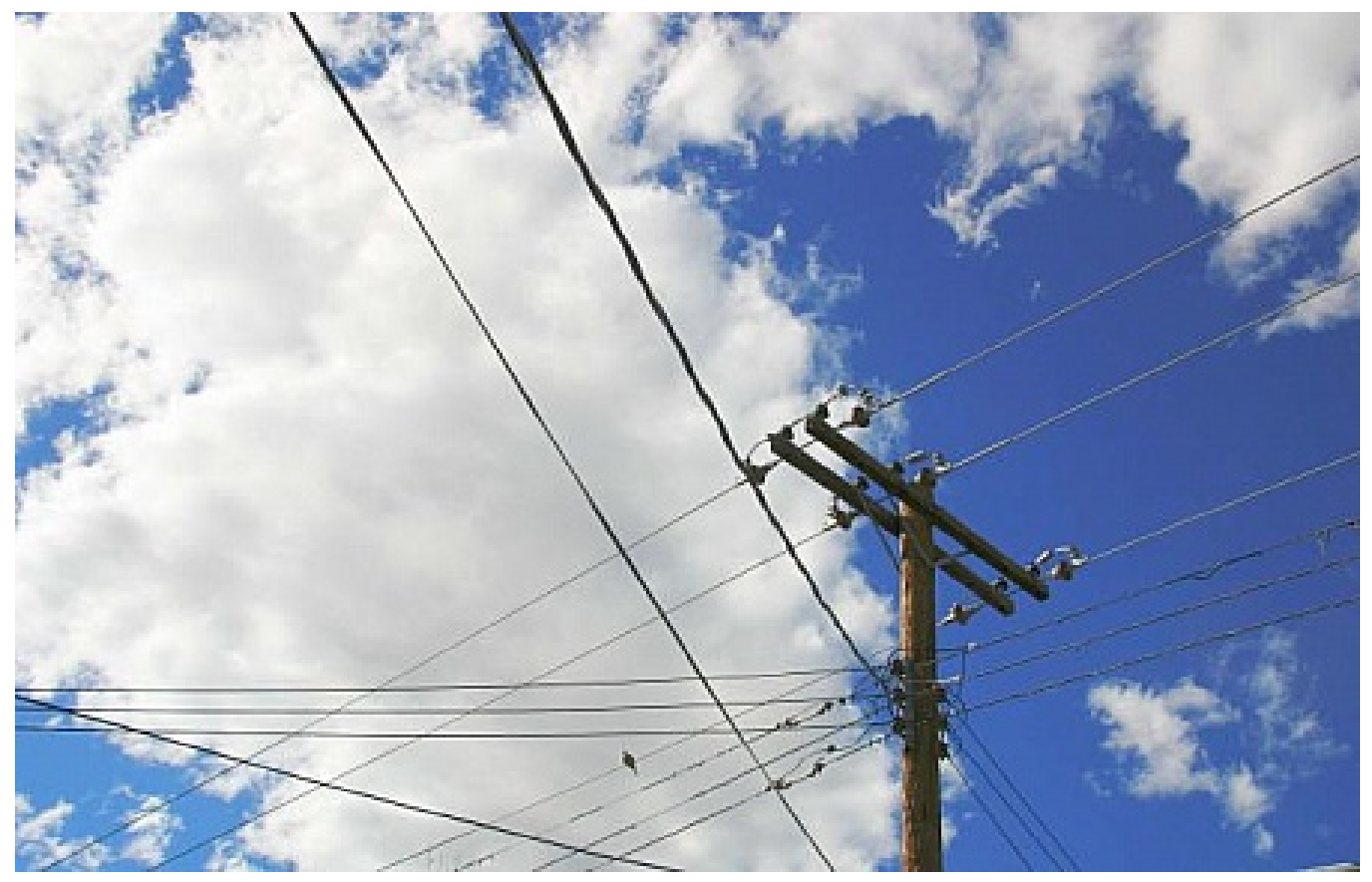

Fuente: https://freerangestock.com/category/65/sky-and-clouds/page1.html 